\title{
Effect of Re content on elastic properties of B2 NiAl from ab initio calculations
}

\author{
A V Ponomareva, Yu Kh Vekilov and Igor Abrikosov
}

\section{Linköping University Post Print}

\section{Tweet}

N.B.: When citing this work, cite the original article.

Original Publication:

A V Ponomareva, Yu Kh Vekilov and Igor Abrikosov, Effect of Re content on elastic properties of B2 NiAl from ab initio calculations, 2014, Journal of Alloys and Compounds, (586), S274-S278.

http://dx.doi.org/10.1016/j.jallcom.2012.12.103

Copyright: Elsevier http://www.elsevier.com/

Postprint available at: Linköping University Electronic Press

http://urn.kb.se/resolve?urn=urn:nbn:se:liu:diva-102967 


\title{
Effect of Re content on elastic properties of B2 NiAl from ab initio calculations
}

\author{
A.V.Ponomareva ${ }^{a}$, Yu.Kh.Vekilov ${ }^{a}$ and I. A. Abrikosov ${ }^{b}$ \\ ${ }^{a}$ Theoretical Physics and Quantum Technology Department, \\ National University of Science and Technology MISIS, RU-119049 Moscow, Russia \\ ${ }^{b}$ Department of Physics, Chemistry and Biology (IFM), \\ Linköping University, SE-581 83 Linköping, Sweden
}

\begin{abstract}
The effect of substitutional alloying of Re on elastic properties of B2 NiAl has been studied using first-principles electronic-structure calculations by the exact muffintin orbitals method and the coherent potential approximation. Our calculations have shown that elastic constants $C_{12}, C_{44}$ and bulk modulus B of $\left(N i_{1-x} R e_{x}\right) A l$ alloys increase with Re composition almost linearly, but concentration dependence of elastic constants $C_{11}$, Young modulus E, shear modulus G, G/B ratio and the Cauchy pressure $P_{C}$ is strongly nonmonotonously and has peculiarities near the concentration $\mathrm{x}=30$ at. \% Re. Analyzing the density of states and Fermi surface sections we establish a direct connection between the behavior of the elastic constants of $\left(N i_{1-x} R e_{x}\right) A l$ alloys and changes in the interatomic bonding and Fermi surface topology.
\end{abstract}

\section{INTRODUCTION}

The intermetallic compound B2 NiAl has high melting temperature (T=1911 K) [1], low density, good oxidation resistance and therefore it widely used in aerospace applications. Low temperature brittleness inherent to $\mathrm{B} 2 \mathrm{NiAl}$ is the main limitation for the use of this intermetallic compounds as a high temperature structural material. Various techniques such as fiber reinforcement, precipitation strengthening, microalloying and macroalloying can improve room-temperature ductility by a formation of specific microstructure [2], [3], [4], [5]. In our previous paper [6] we analyzed substitutional alloying on brittle vs. ductile behavior of $\mathrm{B} 2 \mathrm{NiAl}-\mathrm{X}(\mathrm{X}=\mathrm{Sc}, \mathrm{Ti}, \mathrm{V}, \mathrm{Cr}, \mathrm{W}, \mathrm{Re}, \mathrm{Co})$ alloys examining at atomic level the role of interatomic bonding in the ductility enhancement. We studied the site preference of 
impurities X, elastic constants $C_{11}, C_{12}, C_{44}$, bulk modulus $B$, the Young's modulus $E$, the shear modulus $\mathrm{G}, \mathrm{G} / \mathrm{B}$ ratio and the Cauchy's pressure $\left(P_{C}=C_{12}-C_{44}\right)$ for NiAl-X alloys in the dilute limit, as well as for some nondilute systems to estimate the trend of changes in the elastic properties. Our results demonstrated that Re has a strong preference for the $\mathrm{Ni}$ sublattice in $\mathrm{NiAl}$ in good agreement with available experimental and theoretical data [7], [8].

We predicted that the addition of $\mathrm{W}, \mathrm{V}, \mathrm{Ti}$, and Re atoms could yield improved ductility for B2 NiAl-X alloys without significant changes in the macroscopic elastic moduli. At the same time we found that concentration dependence of $C_{11}$, Young's modulus E, shear modulus $\mathrm{G}, \mathrm{G} / \mathrm{B}$ ratio and the Cauchy pressure $P_{C}$ in $\left(N i_{1-x} R e_{x}\right) A l$ alloys has strongly nonmonotonic character. For this reason we carried out a more detailed study of the effect of Re on elastic properties of B2 NiAl. Investigating the density of states and Fermi surface cross sections we demonstrate that the anomalies in the behavior of the elastic constants have an electronic origin associated with the disappearance of antibonding $e_{g}$ states of nickel.

\section{DETAILS OF CALCULATION}

The calculations were performed using the exact muffin-tin orbital (EMTO) method including the full charge-density technique [9] within the coherent potential approximation (CPA) for modeling substitutionally disordered alloys [10]. The charge density was calculated within the local density approximation (LDA) and the total energy was obtained using the generalized gradient approximation (GGA) [11]. We used a basis set including valence spdf-orbitals and 29x29x29 grid of k-points. The energy integration was carried out in the complex plane using a semi-elliptic contour comprising 24 energy points. In our CPA calculation we included the screening contribution to the electrostatic potential and energy to take into account the effect of charge transfer between the alloy component using screening constants [12] obtained by the locally self-consistent Greens-function (LSGF) method [13]. The calculations of total and projected densities of states (DOS) for B2 $\left(N i_{1-x} R e_{x}\right) A l$ were performed using the Koringa-Kohn-Rostocker (KKR) method within the atomic sphere approximation (ASA) [14], [15]. The Fermi surface cross sections for the alloys were obtained using the peak positions of the Bloch spectral density function (BSD) [16], which was calculated on the grid of k-points lying in ГXMX and XMRM planes of the Brillouin zone. 
For cubic crystals there are three independent elastic constants $C_{11}, C_{12}, C_{44}$ [17]. To determine the tetragonal $\left(C^{\prime}=\left(C_{11^{-}} C_{12}\right) / 2\right)$ and the trigonal $\left(C_{44}\right)$ elastic constants we calculate the internal energy response on a volume-conserving orthorhombic and monoclinic distortions [18] respectively. Then the two cubic elastic constants $C_{11}$ and $C_{12}$ are splitted from the relation between $C^{\prime}$ and bulk modulus $\mathrm{B}=\left(C_{11}+2 C_{12}\right) / 3$. To estimate elastic moduli for polycrystalline materials, the Voigt-Reuss-Hill (VRH) approximation is used [17]. Brittle vs. ductile behavior of alloys is analyzed by using values of the Cauchy's pressure $P_{C}=\left(C_{12}-C_{44}\right)[19]$ and the Pugh's relationship (G/B ratio) [20] phenomenologically linking the plastic properties of materials with their elastic moduli.

If in a material the Cauchy relation $C_{12}=C_{44}$ is fulfilled then the interatomic forces are central-symmetric and the total energy may be reasonably described by the pair potential approximation. But for metals and covalent solids the Cauchy relation is not satisfied experimentally. In these cases we must takes into account the directionality of bonds via many-body potentials [21] and the angular character of atomic bonding relating to the brittle or ductile properties of materials could be described by the Cauchy pressure $P_{C}=\left(C_{12}-C_{44}\right)$ [19]. Therefore for covalent materials where the material resistance to a shear $\left(C_{44}\right)$ is much stronger than a volume change $\left(C_{12}\right)$, Cauchy pressure $P_{C}=\left(C_{12}-C_{44}\right)$ should be negative (vice versa for metallic materials). Another empirical representation which may be considered as a measure of the ductility is $\mathrm{G} / \mathrm{B}$ ratio [20]. Bulk modulus $B$ is associated with the resistance to bond-length change and shear modulus $G$ corresponds to bond-angle change. Therefore, if $G / B<0.5$ a material behaves in a ductile manner, while if $G / B>0.5$ a material should be brittle and the higher the value of $G / B$, the more brittle the material should be. Thus, the use of these physical criteria allows to estimate brittle vs. ductile behavior in alloys and compounds [22], [23].

\section{RESULTS AND DISCUSSION}

Following recipe of Ref. [24] we have established that Re at low concentration should have a strong preference for Ni sublattice [6]. Our results are in good agreement with available experimental data. Indeed, using the atom probe field ion microscopy (APFIM) [7] it was found that Re had a strong preference for the Ni sublattice in NiAl. In principle, a reversal of the site preference could occur with increasing temperature and concentration [6] but as 
demonstrated in Ref. [8] this was more common for additions which did not have any site preference and were randomly distributed between $\mathrm{Al}$ and $\mathrm{Ni}$ sites. So we consider Re only on Ni sublattice.

To analyze alloying effect of Re on the elastic properties of $\mathrm{NiAl}$ we calculate the elastic constants $C_{11}, C_{12}, C_{44}$, Young's modulus E and the shear modulus $G$ of NiAl-Re alloys (Fig. $1)$. It is found that for $\left(N i_{1-x} R e_{x}\right) A l$ alloys the well-known criteria for mechanically stable cubic crystals are satisfied, that is $\left(C_{11}-C_{12}\right)>0, C_{44}>0$. For NiAl the good correlation between our theoretical and experimental lattice parameter, bulk modulus, elastic constants $C_{11}$ and $C_{44}$ is found (Table I). $C_{12}$ obtained in our calculation is slightly smaller than the experimental result so the Pugh ratio $(\mathrm{G} / \mathrm{B})$ is overestimated in comparison with the experimental data. On the contrary Cauchy pressure is underestimated. But though in our calculations NiAl turns out to be "more brittle" than it actually is, we can still analyze and qualitatively estimate the Re additions effect on the ductility of B2 NiAl. For more details see Ref. [6].

From Fig. 1 we see that $C_{12}, C_{44}$ and bulk modulus $B$ are increasing functions at all concentrations although $C_{12}$ and $B$ have a slightly lower rate of increase at low Re concentrations. On the other hand, $C_{11}$ decreases up to a concentration about $30 \%$ of $\operatorname{Re}$ and then substantially increases. The shear modulus $G$ and Young's modulus $E$ almost do not change their values at low Re concentration, and then decrease sharply near 30 at.\% Re and finally increase monotonously up to their original values. The $G / B$ ratio slightly increases and then decreases with increasing rhenium content. The Cauchy pressure $P_{c}$ becomes less positive at low Re concentration and then increases. Thus, using the Pugh criterion and the values of the Cauchy pressure one could expect an increase in ductility of the alloy for Re fraction above 30 at.\%. (or the Re content 15 at.\%.)

In order to understand the reason for the non-monotonous change of elastic properties of NiAl-Re alloys we analyze the electronic density of states (DOS) and Fermi Surface (SF) cross-sections near the singularity. Figs. 2 and 3 show the calculated total and projected DOS for B2 NiAl and NiAl-Re alloys, respectively. From Fig. 2 we can see that the major contribution to DOS in $\mathrm{NiAl}$ around $E_{f}$ comes from narrow d-band of $\mathrm{Ni}$, which consists of the so-called nonbonding [25] $\left(t_{2 g}+e_{g}\right.$ located from -0.25 to $\left.-0.1 \mathrm{Ry}\right)$ and antibonding $\left(e_{g}\right.$ close to $\left.E_{F}\right)$ states, separated by a pseudogap. Note, that the $t_{2 g}\left(d_{x y}, d_{y z}, d_{z x}\right)$ electrons are directed toward the eight nearest neighbors in the bcc lattice, e.g. in $\mathrm{NiAl}$ toward $\mathrm{Al}$ and $\mathrm{Ni}$ 
located on different sublattice of B2 structure. The $e_{g}\left(d_{x^{2}-y^{2}}\right.$ and $\left.d_{z^{2}}\right)$ electrons are directed toward the next-nearest neighbors atoms located on the same sublattice ( $\mathrm{Ni}-\mathrm{Ni}$ and $\mathrm{Al}-\mathrm{Al}$ ). The addition of Re leads to the smearing of total DOS due to the increase of disorder on Ni sublattice (Figure 3, upper panels). The main effect observed at low Re concentration is that the peak corresponding to Ni $e_{g}$ states begins to decrease (Fig. 3, $\left(R e_{10} N i_{90}\right) A l$ and $\left.\left(R e_{20} N i_{80}\right) A l\right)$ and at about 30 at.\% Re it disappears (Fig. 3, $\left.\left(R e_{30} N i_{70}\right) A l\right)$. Simultanously, $C_{11}$ decreases and reaching its minimum causes a drop in $G$ and $E$ moduli. In the same concentration interval the Pugh criterion and the values of the Cauchy pressure indicate a possibility for a slight decrease in ductility. Also, this indicates that the reduction of the Ni concentration increases a partial covalent component in the bonding, although the metallic type of bond still dominates $\left(C_{12}>C_{44}\right.$, Fig. 1). A small dip in $P_{c}$ curve is also related to the decrease of occupation of $\mathrm{Ni} e_{g}$ states. Indeed in $\mathrm{B} 2 \mathrm{NiAl} t_{2 g}$ and $e_{g}$ states are almost fully occupied (Fig. 2), and therefore there is no specific directionality in the distribution of electron density. In $\left(R e_{x} N i_{1-x}\right) A l$ at low $\mathrm{x} e_{g}$ states of $\mathrm{Ni}$ start to disappear. Thus electron density distribution becomes more directional. With further increase of Re concentration, delectrons of Re, which occupy a wide band and have large spatial extension begin to provide an increase of the bond strength. So $C_{11}$, the shear modulus $G$ and Young's modulus $E$ start to increase. We can see that with increasing Re concentration central peak is smeared out, broadens and shifts to the right as compared to the NiAl case. Such delocalization of electrons increases metallic component of bonding at $\mathrm{x}>30$ at.\% Re. At the same time, a type of interactions remains mixed: the increasing value of $C_{44}$ indicates the presence of covalent component of bonding. We can see strong hybridization between $t_{2 g}$ states of Re and p-electrons of $\mathrm{Al}$ which is much stronger that in the case of d-electrons of nickel. This strengthens covalent component of bonding and enhances the shear resistance.

Finally in Figure 4 we present fragments of Fermi surface cross sections by ГXMX and XMRM planes in $\left(R e_{x} N i_{1-x}\right) A l$ alloys with $\mathrm{x}=0,10,20,30,50$ at.\% Re. The figures allow us to see the evolution of the Fermi surface (FS) topology with increasing Re concentration. The ГXMX and XMRM sections of the Fermi surface show a hole pocket at the center of the zone and electron sheets around the perimeter (Fig.4, ГXMX plane, upper panel), as well as small fairly spherical hole pockets around R points of the Brillouin zone (BZ) (Fig.4, XMRM plane, lower panel). At small Re concentration peaks of Bloch spectral function (BSF) are smeared out and the electronic parts of the Fermi surface sheets become smaller 
(Fig.4, $\left.\left(R e_{10} N i_{90}\right) A l\right)$. At $\mathrm{x}=20$ at. \% Re the sheet touches the BZ boundary near the $\mathrm{X}$ point forming a neck (Fig.4, $\left.\left(R e_{20} N i_{80}\right) A l\right)$. Also in the FS of $\left(R e_{20} N i_{80}\right) A l$ a formation of a new electron sheet around the $\Gamma$ point begins. At $\mathrm{x}=30$ at. $\%$ Re, when $e_{g}$ peak of the DOS vanishes, a part of the original electronic sheet near XM line disappears in ГXMX plane, and only the electronic pockets around the M point remain. At the same time a new cavity near the $\Gamma$ point with arms along $\Gamma X$ direction grows. So changes in the topology of Fermi surface take place also near 30 at. \% of Re.

\section{CONCLUSION}

We have investigated effect of rhenium content on the elastic properties and on ductility enhancement of $\left(N i_{1-x} R e_{x}\right) A l$ alloys. We have shown that singularities of DOS and BSF peaks are observed at the same concentrations as the peculiarities in the behavior of the elastic properties of NiAl-Re alloys. We have linked this correlation with the disappearance of antibonding $e_{g}$ states of nickel at low Re concentration. Metallic-covalent (mixed) bonding in the alloys together with a growing metallic component with increasing Re concentration leads to the fact that all three elastic constants $C_{11}, C_{12}, C_{44}$ increase at $x>30$. Compared with elastic constants of $\mathrm{B} 2 \mathrm{NiAl} C_{11}, C_{12}, C_{44}$ increase by about $15 \%, 25 \%$ and $50 \%$, respectively. Analyzing the calculated values of the Cauchy pressure and the Pugh criterion one can expect an increase in the ductility of the alloy for Re fraction beyond 30 at.\%.

\section{ACKNOWLEDGEMENTS}

Financial support from RFBR (Grant \# 10-02-00-194a), from the Ministry of Education and Science of the Russian Federation within the framework of Program "Research and Pedagogical Personnel for Innovative Russia (2009-2013)" (project no. 14.37.21.0890 of 10.09.2012), as well as from the Swedish Foundation for Strategic Research (SSF) program SRL10-0026 is acknowledged. Calculations are performed on the Joint Supercomputer Center of RAS (Moscow, Russia) and National Supercomputer Centre (NSC) in Linköping, 
Sweden.

[1] T.B. Massalski, J.L. Murray, L.H. Bennett and H. Baker, Binary Alloy Phase Diagrams, American Society for Metals,Metals Park, OH, (1986).

[2] I.Baker and P.R.Munroe, Improving Intermetallic Ductility and Toughness, J.Met. 40 (1998) $28-31$.

[3] K.Ishida, R.Kainuma, N.Ueno and T. Nishizawa, Ductility enhancement in NiAl B2-base alloys by microstructural control, Metallurgical and Materials Transactions A 22 (1991) 441-446.

[4] G. Frommeyer, R.Rablbauer, H.J.Schäfer, Elastic properties of B2-ordered NiAl and NiAl-X (Cr, Mo, W) alloys, Intermetallics 18 (2010) 299-305.

[5] H. Li, J. Guo, H.Ye, Q. Wang and J.He, Simultaneous improvement of strength and ductility in NiAl-Cr(Mo)-Hf near eutectic alloy by small amount of Ti alloying addition, Mater. Lett. $62(2008) 61-64$.

[6] A.V.Ponomareva, E.I.Isaev, Yu.Kh.Vekilov and I.A Abrikosov, Site preference and effect of alloying on elastic properties of ternary B2 NiAl-based alloys, Phys. Rev. B 85 (2012) 144117-1 $-144117-10$.

[7] G. Frommeyer, R. Fischer, J. Deges, R. Rablbauer, A. Schneider, APFIM investigations on site occupancies of the ternary alloying elements Cr, Fe, and Re in NiAl, Ultramicroscopy 101 (2004) 139-148.

[8] C.Jiang, Site preference of transition-metal elements in B2 NiAl: A comprehensive study, Acta Mater. 55 (2007) 4799-4806.

[9] L. Vitos, Computational Quantum Mechanics for Materials Engineers: The EMTO Method and Applications Springer-Verlag, London, (2007).

[10] L. Vitos, I. A. Abrikosov, and B. Johansson, Anisotropic Lattice Distortions in Random Alloys from First-Principles Theory, Phys. Rev. Lett. 87 (2001) 156401-1-156401-4.

[11] J.P. Perdew, K. Burke and M. Ernzerhof, Generalized gradient approximation made simple, Phys. Rev. Lett. 77 (1996) 3865.

[12] A.V.Ruban and H.L.Skriver, Screened Coulomb interactions in metallic alloys. I. Universal screening in the atomic-sphere approximation, Phys. Rev. B 66 (2002) 024201; A.V.Ruban, S.I.Simak, P.A.Korzhavyi and H.L.Skriver, Screened Coulomb interactions in metallic alloys. 
II. Screening beyond the single-site and atomic-sphere approximations, Rhys. Rev. B 66 (2002) 024202 .

[13] I.A. Abrikosov, A.M.N. Niklasson, S.I. Simak, B. Johansson, A.V. Ruban, and H.L. Skriver, Order- N Green's Function Technique for Local Environment Effects in Alloys, Phys. Rev. Lett. 76 (1996) 4203; I.A.Abrikosov, S. I. Simak, B. Johansson, A. V. Ruban, and H.L. Skriver, Locally self-consistent Green's function approach to the electronic structure problem, Phys. Rev. B 56 (1997) 9319.

[14] H. L. Skriver and N. M. Rosengaard, Self-consistent Green's-function technique for surfaces and interfaces, Phys. Rev. B 43 (1991) 9538.

[15] I. A. Abrikosov and H. L. Skriver, Self-consistent linear-muffin-tin-orbitals coherent-potential technique for bulk and surface calculations: $\mathrm{Cu}-\mathrm{Ni}, \mathrm{Ag}-\mathrm{Pd}$, and $\mathrm{Au}-\mathrm{Pt}$ random alloys, Phys. Rev. B 47 (1993) 16532.

[16] Ya.M. Blanter, M. I. Kaganov, A.V. Pantsulaya and A.A. Varlamov, The theory of electronic topological transitions, Phys. Rep. 245 (1994) 159.

[17] G.Grimvall, Thermophysical Properties of Materials, 1st Edition. Elsevier, (1999)

[18] M.J.Mehl, J.E.Osburn, D.A.Papaconstantopoulos, and B.M.Klein, Structural properties of ordered high-melting-temperature intermetallic alloys from first-principles total-energy calculations, Phys. Rev. B 41 (1990) 10311.

[19] D. Pettifor, Theoretical predictions of structure and related properties of intermetallics, Mater. Sci. Tech. 8 (1992) 345.

[20] S.F. Pugh, Relations between the elastic moduli and the plastic properties of polycrystalline pure metals, Philos. Mag. 45 (1954) 823.

[21] E.G.Brovman, Yu.M. Kagan, Phonons in nontransition metals, Sov. Phys. Usp. 17 (1974) 125.

[22] G.N Greaves, A.L.Greer, R.S. Lakes and T.Rouxel, Poisson's ratio and modern materials, Nature Materials, 10 (2011), 823.

[23] D. G. Sangiovanni, V. Chirita, and L. Hultman, Electronic mechanism for toughness enhancement in $T i_{x} M_{1-x} N(\mathrm{M}=$ Mo and W), Phys. Rev. B 81 (2010) 104107 (2010).

[24] A.V.Ruban and H.L.Skriver, Calculated site substitution in ternary gamma(prime)-Ni3Al: Temperature and composition effects, Phys. Rev.B. 55 (1997) 856.

[25] P. Mohn, C. Persson, P. Blaha, K. Schwarz, P. Novak, and H. Eschrig, Correlation Induced Paramagnetic Ground State in FeAl, Phys. Rev. Lett. 87 (2001) 196401. 
[26] G. Simmons, H. Wang. Single Crystal Elastic Constants and Calculated Aggregate Properties: A Handbook. MIT Press, Cambridge, MA, USA (1971), 370. 
FIG. 1: (Color online) Calculated elastic constants $C_{11}, C_{12}, C_{44}$, Pugh G/B ratio, Cauchy pressure $P_{C}=\left(C_{12}-C_{44}\right)$, shear moduli $G$, bulk moduli $B$, and Young's moduli $E$ of NiAl-Re alloys.

FIG. 2: (Color online) Calculated total and projected densities of states for B2 NiAl. The Fermi level is set to zero.

TABLE I: Young's modulus $(E)$, shear modulus $(G)$ of B2 NiAl. The experimental values for NiAl [26] are given in parentheses.

\begin{tabular}{|c|c|c|c|c|c|c|}
\hline $\mathrm{a}, \AA$ & $C_{11}, \mathrm{GPa}$ & $C_{12}, \mathrm{GPa}$ & $C_{44}, \mathrm{GPa}$ & $\mathrm{B}, \mathrm{GPa}$ & $\mathrm{E}, \mathrm{GPa}$ & $\mathrm{G}, \mathrm{GPa}$ \\
\hline 2.89 & 233 & 121 & 114 & 159 & 218 & 86 \\
$(2.89)$ & $(212)$ & $(143)$ & $(112)$ & $(166)$ & $(184)$ & $(70)$ \\
\hline
\end{tabular}


FIG. 3: (Color online) Calculated total and projected densities of states for B2 $\left(R e_{x} N i_{1-x}\right) A l$ alloys. Shadowed area correspond to DOS of B2 NiAl.

FIG. 4: (Color online) $\Gamma X M X$ and XMRM sections of Fermi surface in $\left(R e_{x} N i_{1-x}\right) A l$ alloys with $\mathrm{x}=0,10,20,30,50$ at. $\%$ Re. 
$\left(\mathrm{Ni}_{1-\mathrm{x}} \mathrm{Re}_{\mathrm{x}}\right) \mathrm{Al}$
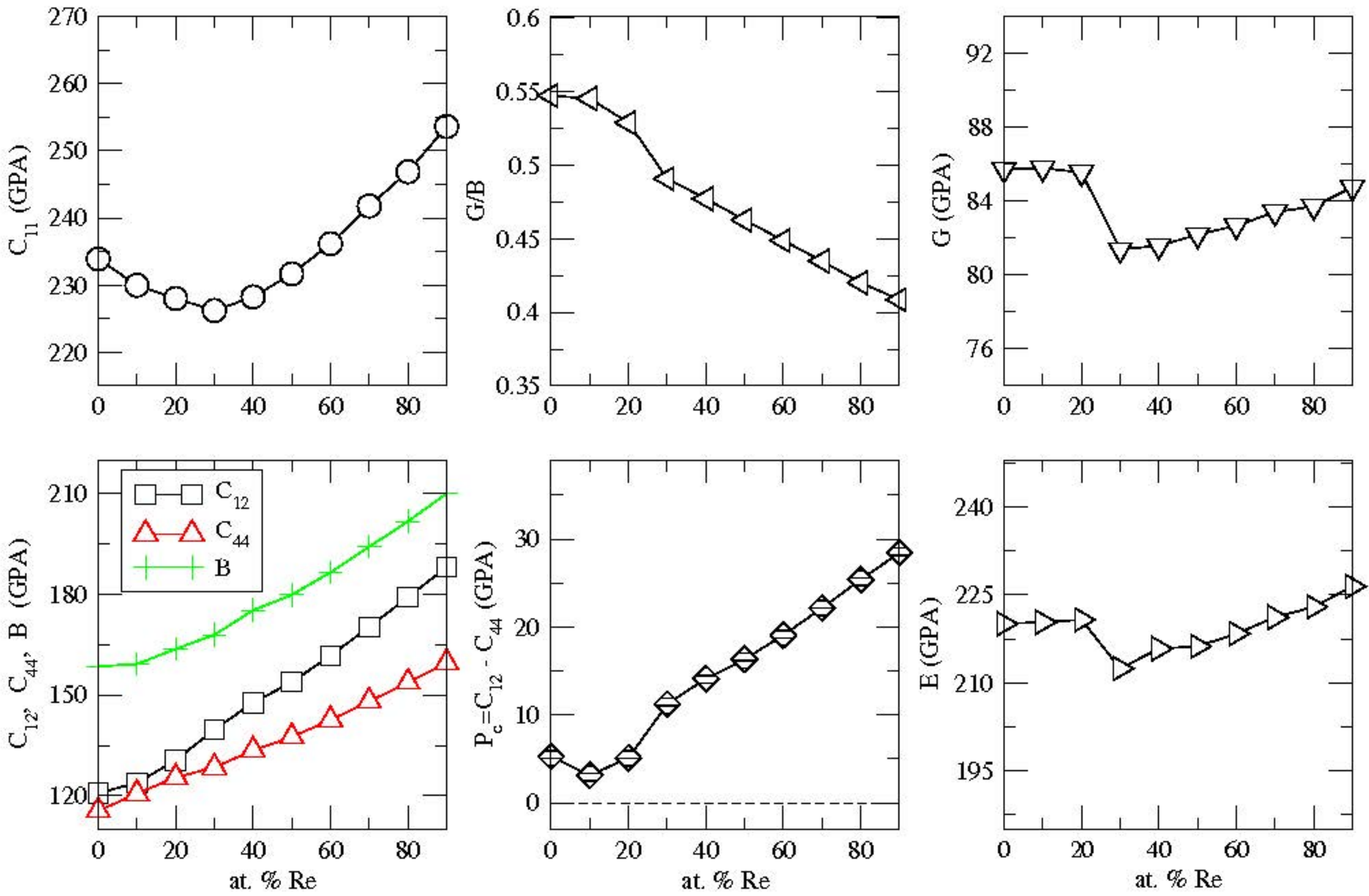


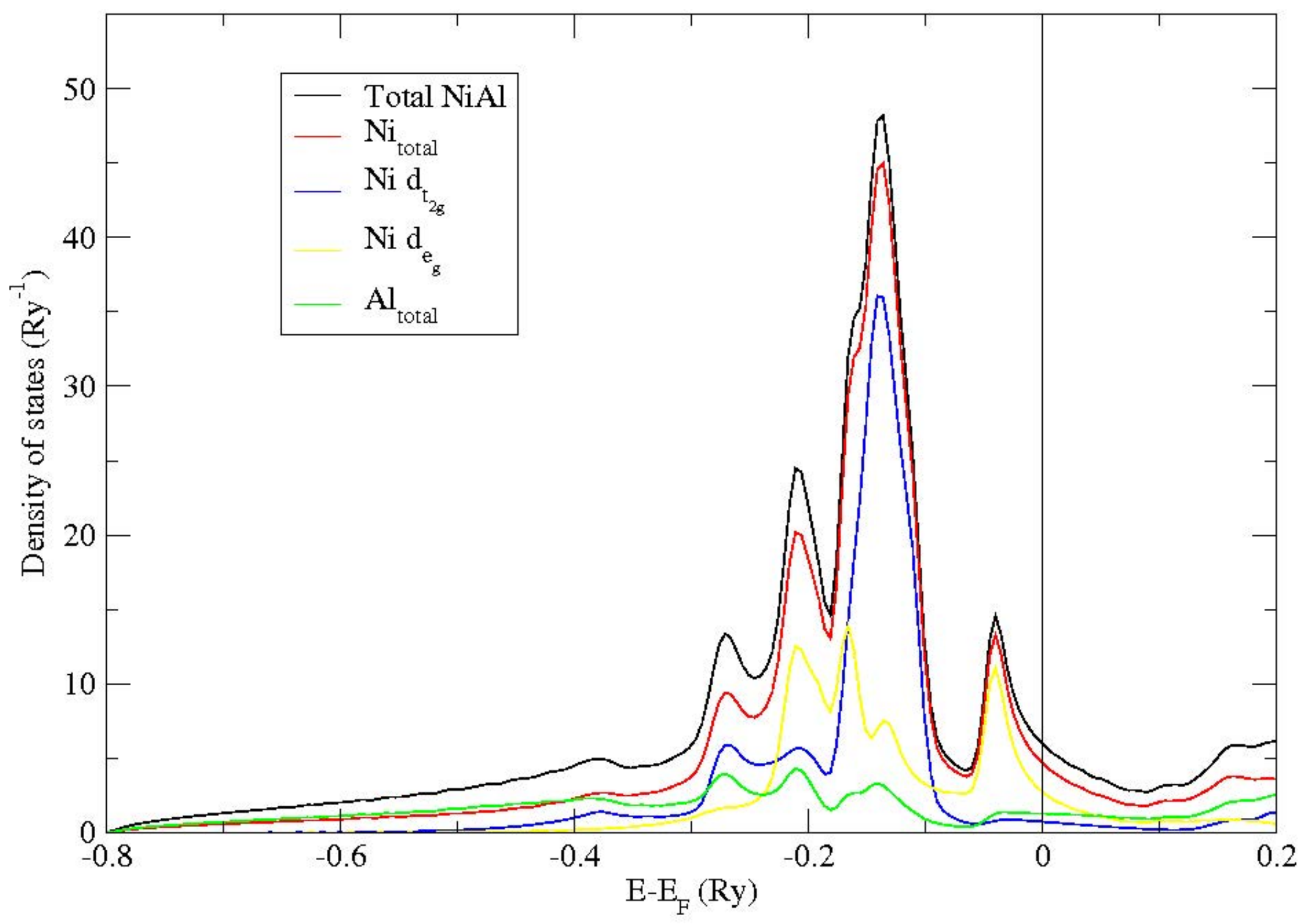




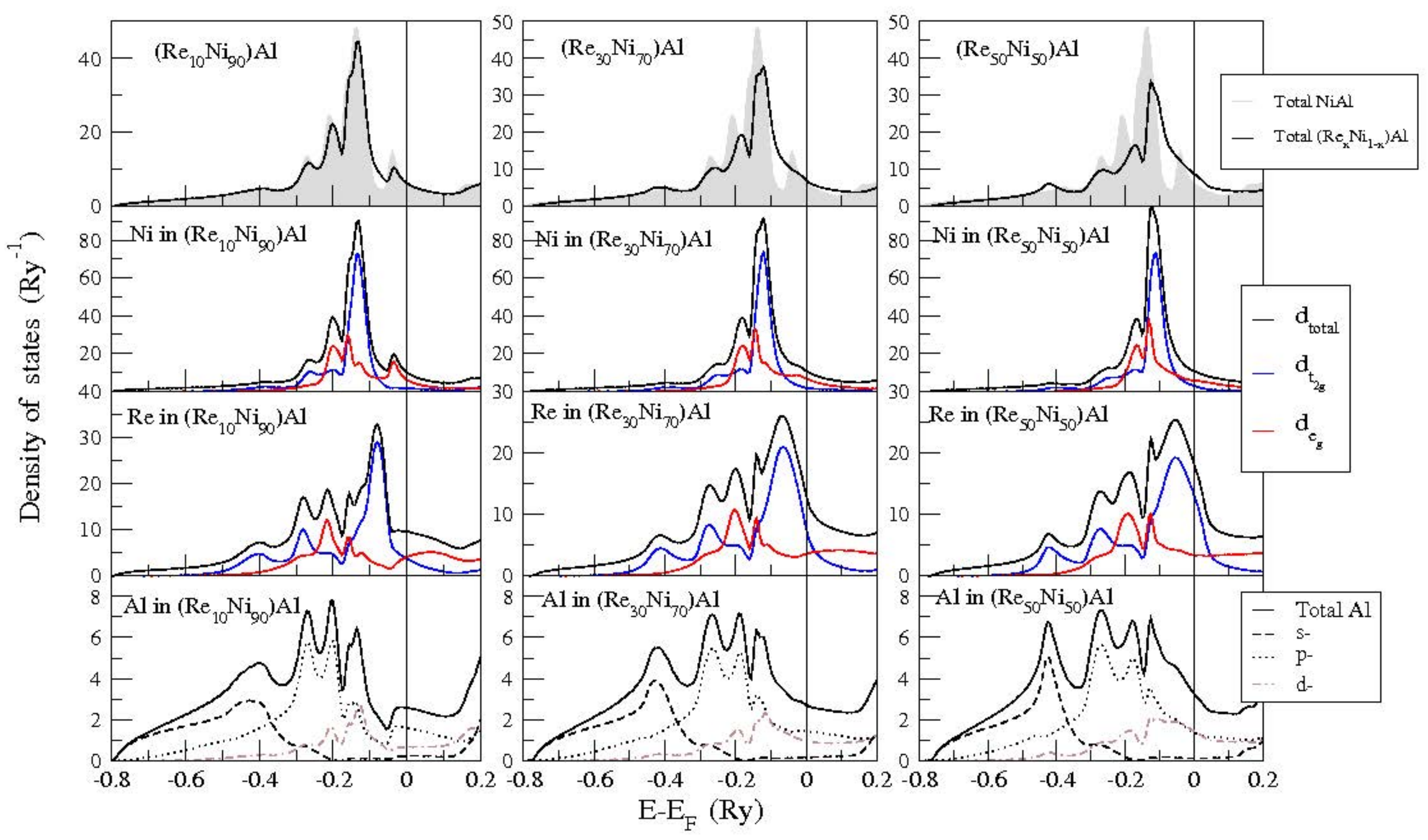


NiAL

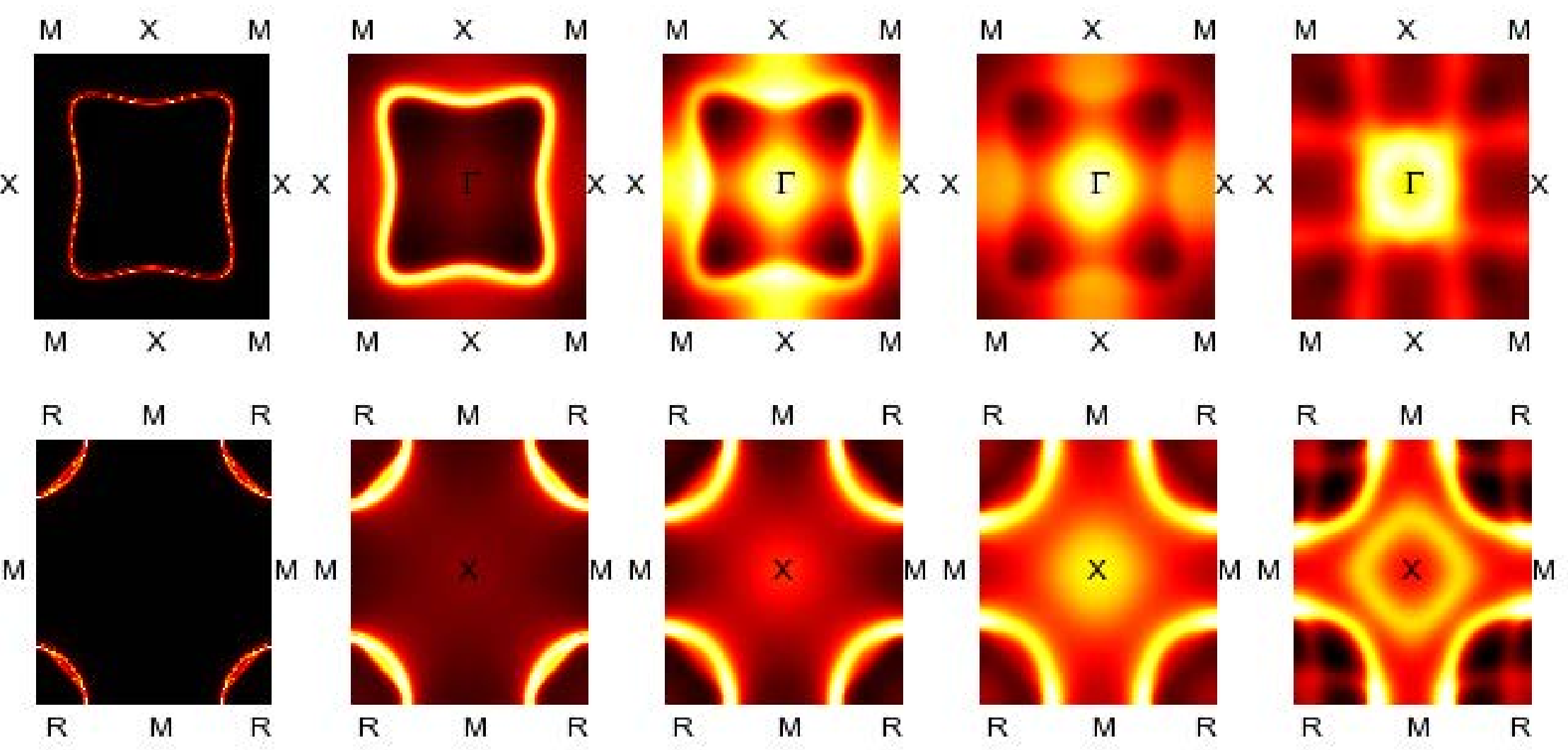

